\title{
PROCESO SALUD-ENFERMEDAD EN LA REGIÓN AMAZÓNICA: FACTORES AMBIENTALES Y LA APARICIÓN DE ENFERMEDADES
}

\section{ARTÍCULO DE REVISIÓN}

CUSTÓDIO, Wenderson Picanço ${ }^{1}$, CHAVES, Juliana Vitória Rocha Leite ${ }^{2}$, PANTOJA, Patrícia Trindade ${ }^{3}$, CÁRDENAS, Anneli Mercedes Celis de ${ }^{4}$, ANDRADE, Rosemary Ferreira de ${ }^{5}$, DAPUREZA, Demilto Yamaguchi ${ }^{6}$, FECURY, Amanda Alves ${ }^{7}$ \section{CUSTÓDIO, Wenderson Picanço. Et al. Proceso salud-enfermedad en la región} amazónica: factores ambientales y la aparición de enfermedades. Revista Científica Multidisciplinar Núcleo do Conhecimento. Año. 06, Ed. 12, Vol. 03, pp. 0521. Diciembre de 2021. ISSN: 2448-0959, Enlace de acceso: https://www.nucleodoconhecimento.com.br/salud/aparicion-de-enfermedades, DOI: 10.32749/nucleodoconhecimento.com.br/salud/aparicion-de-enfermedades

\section{RESUMEN}

\footnotetext{
${ }^{1}$ Magíster en Ciencias de la Salud de la Universidad Federal de Amapá; Especialista en Hematología por la Unión Brasileña de Colegios; Licenciado en Biomedicina por la Facultad de Macapá; Graduado en Pedagogía por la Universidad de la Ciudad de São Paulo; Graduado en Licenciatura en Ciencias Biológicas Ciudad Universitaria de São Paulo. ORCID: 0000-0003-0424-6852.

${ }^{2}$ Estudiante de Maestría en Ciencias de la Salud de la Universidad Federal de Amapá; Postgrado en Odontología Pediátrica por el Grupo de Especialidades Dentales; Postgrado en Odontología para pacientes con cáncer por el Hospital Sirio Libanés; Licenciado en Odontología por la Facultad de Macapá - FAMA. ORCID: 0000-0002-7595-9094.

${ }^{3}$ Estudiante de Maestría en Ciencias de la Salud de la Universidad Federal de Amapá; Postgrado en Urgencias y Emergencias y UCl por el Colegio Madre Tereza-FAMAT; Postgrado en Docencia y Gestión de la Educación Superior por Fatec College; Graduado en Enfermería de la Facultad de Macapá - FAMA. ORCID: 0000-00028667-0165.

${ }^{4}$ Doctor en Enfermería, Escuela de Enfermería, Universidad de São Paulo EEUSP, Maestría en Enfermería Fundamental, Universidad de São Paulo EEUSP. Graduado en Enfermería por la Universidad Nacional del Callao en Lima Perú. ORCID: https://orcid.org/0000-0002-6581-4326.

${ }^{5}$ Graduado en Enfermería y Obstetricia por la Universidad Estatal de Pará (UEPA), Magíster en Enfermería (UFPA) y Doctor en Ciencias: desarrollo socioambiental (NAEA/UFPA). Actualmente es profesor titular en la Universidad Federal de Amapá. Desarrolla trabajos sobre los siguientes temas: salud pública, epidemiología, amapá, Amazonía, malaria y migración. ORCID: https://orcid.org/0000-0003-4472-8565.

${ }^{6}$ Doctor en Ciencias, Máster en Educación Física y Graduado en Educación Física. ORCID: https://orcid.org/0000-0001-8336-2178.

${ }^{7}$ Doctor y Máster en Enfermedades Tropicales, Especialista en Microbiología, Biomédica ORCID: http://orcid.org/0000-0001-5128-8903.
}

$\mathrm{RC}: 104516$

Lien d'accès: https://www.nucleodoconhecimento.com.br/sante/emergence-de-maladies 
Introducción: La Amazonía siempre ha sido objeto de numerosos estudios centrados en su entorno, sociedad y salud. Pregunta de la guía: ¿Qué factores ambientales contribuyen a la aparición de enfermedades en la región amazónica? Objetivo: Abordar los principales factores ambientales que contribuyen a la aparición de enfermedades en la región amazónica. Método: Se trata de una revisión de la literatura en las bases de datos Scientific Electronic Library Online (SciELO), US National Library of Medicine National Institutes of Health (PubMeD), la Biblioteca Virtual en Salud (BVS) y el Instituto Nacional de Investigaciones Espaciales (INPE). En total, se utilizaron 18 artículos en la construcción del estudio, todos los cuales pasaron por los criterios de inclusión y exclusión establecidos para filtrar solo revistas con enfoques temáticos. Resultados: Desde el punto de vista epidemiológico, el espacio amazónico formado por la interacción del sistema socioecológico tiene características diferentes a las de otras partes del país. Esta diferencia es causada por la base ecológica natural y sus formas de ocupación y desarrollo. Conclusión: Los principales factores ambientales que contribuyen a la aparición de enfermedades en la región amazónica son la deforestación, la quema, la contaminación de los ríos, el proceso de urbanización y la agroindustria.

Palabras clave: Salud-enfermedad, Amazonía, Factores ambientales.

\section{INTRODUCCIÓN}

La Amazonía siempre ha sido objeto de numerosos estudios centrados en su entorno, sociedad y desarrollo. Las publicaciones de las últimas décadas han discutido importantes aspectos regionales como la biodiversidad, los usos de la tierra, la ciencia y la tecnología, el crecimiento urbano (BROWDER y GODFREY, 2017), el desarrollo sostenible y los proyectos de desarrollo que causan deforestación (PERZ, 2002).

Para entender la complejidad de la Amazonía en relación a las diferentes regiones del mundo, es importante recordar que esta complejidad no se limita a sus características biológicas y ambientales, sino que está relacionada con la forma en que la población que la habita difiere en su forma de vida y en la forma de actuar y pensar (ROLIM, 2015).

$\mathrm{RC}: 104516$

Lien d'accès: https://www.nucleodoconhecimento.com.br/sante/emergence-de-maladies 
El concepto de salud-enfermedad puede asumir una dimensión regional, ya que en esta región existe un inmenso vacío de información científica sobre las condiciones sociales y ambientales, para satisfacer las demandas de la sociedad. Cabe mencionar que en esta región existe una alta concentración de personas fijadas en los centros urbanos, especialmente en las capitales, causando problemas generalizados en los grandes centros como: falta de agua tratada y saneamiento básico, proliferación de insectos vectores de enfermedades, generalmente anofelina transmisora de malaria, dengue entre otros (CAÑETE y RAVENA-CAÑETE, 2010).

La elección de esta región como objeto de análisis se justifica por su diversidad ambiental (clima, ecosistemas y biodiversidad) y demográfica (diversidad social con baja densidad de población y concentración en las ciudades), que, en combinación con procesos económicos como la agricultura extensiva, la ganadería, la explotación minera, la hidroelectricidad y la industria impactan en la salud de la población según su modelo de desarrollo. En base a esto, la pregunta principal del estudio es: ¿qué factores ambientales contribuyen a la aparición de enfermedades en la región amazónica?

Por lo tanto, el objetivo de este estudio fue resaltar los factores ambientales que contribuyen a la aparición de enfermedades en la región amazónica, así como enfatizar la investigación dirigida a la región, lo que permitió una mayor colección bibliográfica sobre el tema, además de resaltar la necesidad de creación y reformulación de políticas de salud pública para la población que vive en esta región.

\section{METODOLOGÍA}

Este trabajo se realizó a través de una revisión bibliográfica en revistas científicas, disponibles en los sitios web y bases de datos: Scielo,pubmed, Biblioteca Virtual en Salud y estudios realizados por el Instituto Nacional de Investigaciones Espaciales. Se realizaron búsquedas en los artículos entre agosto y noviembre de 2021, y se recopilaron 18 artículos y se seleccionaron todos ellos para la construcción del estudio. Los criterios de inclusión fueron artículos publicados entre 2000 y 2021 , en inglés, español y portugués. Por otro lado, los criterios de exclusión fueron artículos RC: 104516

Lien d'accès: https://www.nucleodoconhecimento.com.br/sante/emergence-de-maladies 
publicados antes del año 2000, duplicados, editoriales de la carta del editor, así como revistas en idiomas diferentes a los mencionados en los criterios de inclusión. Los descriptores utilizados para la búsqueda en los estudios fueron: Salud-enfermedad; Amazonía; Factores ambientales.

Por lo tanto, este artículo es un análisis del proceso salud-enfermedad en la región amazónica y los factores ambientales que influyen en la aparición de enfermedades (factores sociales, económicos, culturales y ambientales, y otros factores que afectan la ocurrencia de problemas de salud en la población), según Sobral y Freitas (2010) utilizando variables cuantificables (indicadores) y revisión de la literatura.

\section{RESULTADOS}

Tabla 1. Principales resultados de artículos seleccionados para la investigación.

\begin{tabular}{|c|c|c|c|}
\hline & Título & $\begin{array}{l}\text { Autores, año } \\
\text { de } \\
\text { publicación } \\
\text { y país }\end{array}$ & Resultados principales \\
\hline ARTÍ́CULO & $\begin{array}{l}\text { A evolução do } \\
\text { conhecimento sobre } \\
\text { hepatites virais na } \\
\text { região Amazônica: da } \\
\text { epidemiologia e } \\
\text { etiologia à prevenção. }\end{array}$ & $\begin{array}{l}\text { Bensabath e } \\
\text { Soares, } \\
\text { 2016, Brasil. }\end{array}$ & $\begin{array}{l}\text { El estudio busca demostrar } \\
\text { una enfermedad similar a la } \\
\text { fiebre amarilla, pero } \\
\text { considerada como una } \\
\text { enfermedad nueva, ocurre en } \\
\text { zonas de los valles de los ríos } \\
\text { Juruá, Purús y Madeira. } \\
\text { Temida por los residentes por } \\
\text { su alta letalidad, siendo } \\
\text { clínicamente } \\
\text { hepatoencefalopatía } \\
\text { evolución fulminante (media } \\
\text { de } 5 \text { a } 6 \text { días). }\end{array}$ \\
\hline ARTÍCULO & Chagas disease and & Briceño- & El artículo \\
\hline
\end{tabular}

$\mathrm{RC}: 104516$

Lien d'accès: https://www.nucleodoconhecimento.com.br/sante/emergence-de-maladies 


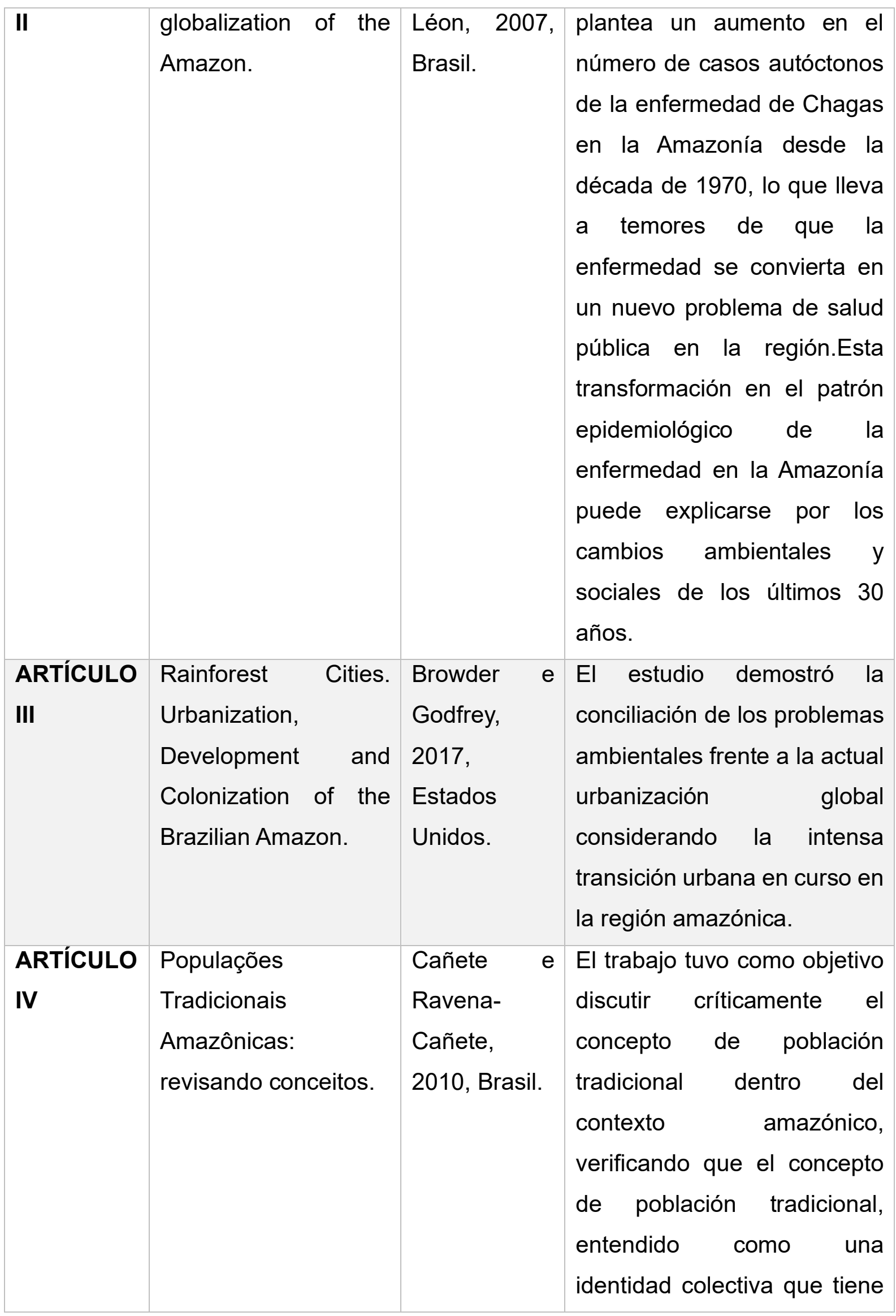

RC: 104516

Lien d'accès: https://www.nucleodoconhecimento.com.br/sante/emergence-de-maladies 


\begin{tabular}{|c|c|c|c|}
\hline & & & $\begin{array}{l}\text { la especificidad de desarrollar } \\
\text { formas de vida de integración } \\
\text { e intimidad con el medio } \\
\text { ambiente. }\end{array}$ \\
\hline $\begin{array}{l}\text { ARTÍCULO } \\
\text { v }\end{array}$ & $\begin{array}{lr}\text { O trabalho como } \\
\text { determinante } \\
\text { processo } \\
\text { doença. }\end{array}$ & $\begin{array}{l}\text { Cardoso, } \\
\text { 2015, Brasil. }\end{array}$ & $\begin{array}{l}\text { El estudio analizó cómo, en el } \\
\text { contexto actual, las } \\
\text { condiciones organizativas y } \\
\text { físicas de trabajo, situaciones } \\
\text { y relaciones laborales y } \\
\text { formas de gestión están } \\
\text { determinando } \\
\text { contribuyendo a la } \\
\text { enfermedad de los } \\
\text { trabajadores para entender } \\
\text { una relación tan compleja. }\end{array}$ \\
\hline $\begin{array}{l}\text { ARTÍ́CULO } \\
\text { VI }\end{array}$ & $\begin{array}{l}\text { Determinante da } \\
\text { saúde no Brasil: a } \\
\text { procura da equidade } \\
\text { na saúde. }\end{array}$ & $\begin{array}{l}\text { Carrapato et } \\
\text { al., } 2017, \\
\text { Brasil. }\end{array}$ & $\begin{array}{l}\text { El presente estudio tuvo } \\
\text { como objetivo identificar, a } \\
\text { través de la bibliometría, } \\
\text { cuáles son los determinantes } \\
\text { de la salud con mayor } \\
\text { impacto en la salud de la } \\
\text { población ante el tema de que } \\
\text { la calidad de vida se asocia } \\
\text { casi de inmediato con la } \\
\text { salud. }\end{array}$ \\
\hline $\begin{array}{l}\text { ARTÍCULO } \\
\text { VII }\end{array}$ & $\begin{array}{l}\text { The Health of } \\
\text { Indigenous Peoples in } \\
\text { the Brazilian Amazon. }\end{array}$ & $\begin{array}{l}\text { Confalonieri, } \\
2000, \\
\text { Amsterdam. }\end{array}$ & $\begin{array}{l}\text { El artículo buscó demostrar } \\
\text { las enfermedades } \\
\text { epidémicas y la destrucción } \\
\text { ambiental de muchas tribus } \\
\text { que han pasado del contacto } \\
\text { al desplazamiento, la } \\
\text { aniquilación y la extinción en }\end{array}$ \\
\hline
\end{tabular}

RC: 104516

Lien d'accès: https://www.nucleodoconhecimento.com.br/sante/emergence-de-maladies 


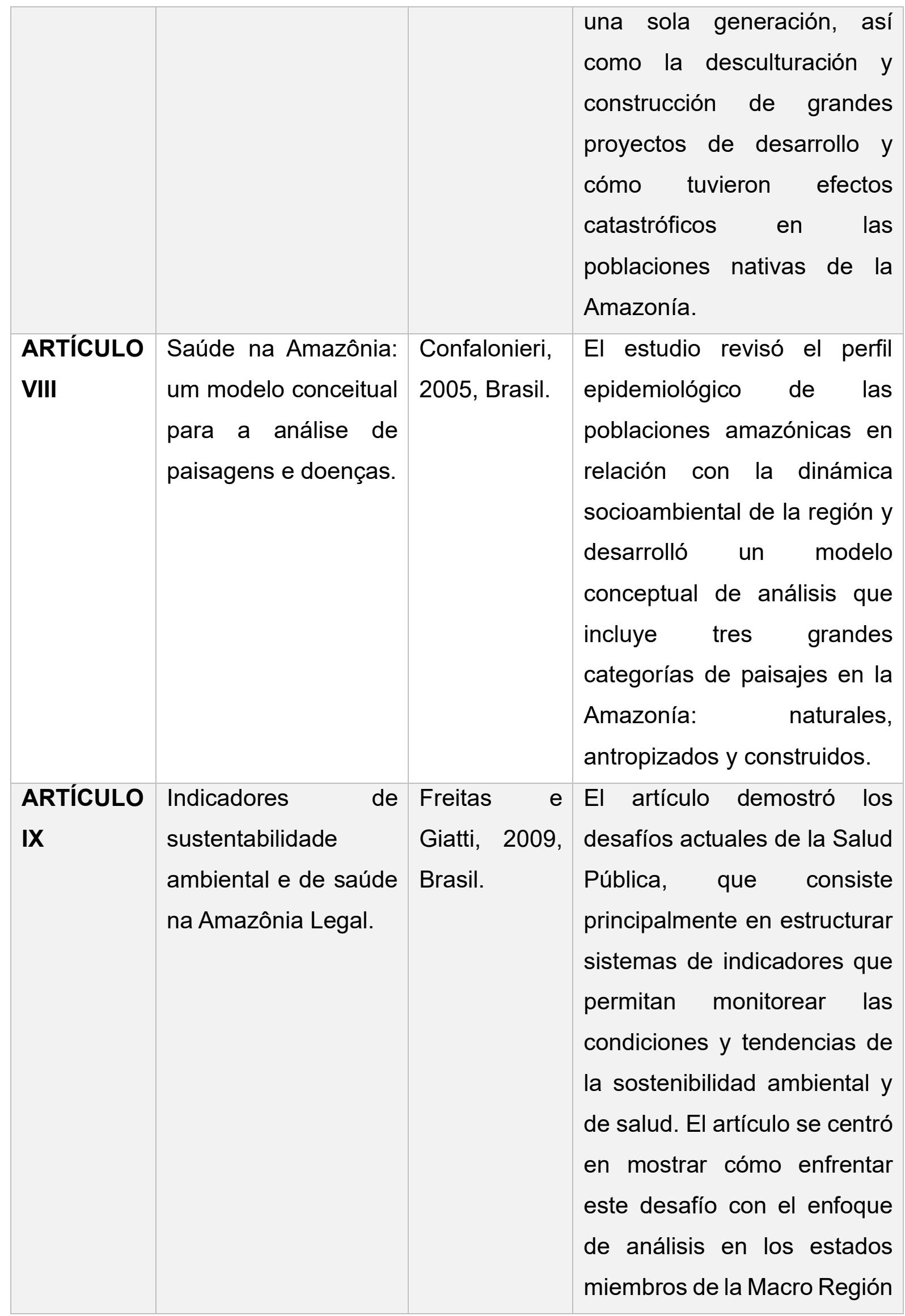

RC: 104516

Lien d'accès: https://www.nucleodoconhecimento.com.br/sante/emergence-de-maladies 


\begin{tabular}{|c|c|c|c|}
\hline & & & Amazónica Legal. \\
\hline $\begin{array}{l}\text { ARTíCULO } \\
\mathbf{x}\end{array}$ & $\begin{array}{l}\text { Custo das doenças } \\
\text { atribuíveis a fatores } \\
\text { ambientais na cidade } \\
\text { de Manaus, } \\
\text { Amazonas, Brasil. }\end{array}$ & $\begin{array}{l}\text { Medeiros, } \\
\text { 2014, Brasil. }\end{array}$ & $\begin{array}{l}\text { El estudio presentó la } \\
\text { estimación del costo de las } \\
\text { enfermedades atribuibles a } \\
\text { factores ambientales en la } \\
\text { ciudad de Manaos, entre } \\
1998 \text { y 2009, mostró las } \\
\text { causas de hospitalización y } \\
\text { cómo se agruparon en base a } \\
\text { los estudios de Carga Global } \\
\text { de Enfermedad y análisis } \\
\text { comparativo de riesgos de la } \\
\text { Organización Mundial de la } \\
\text { Salud. }\end{array}$ \\
\hline $\begin{array}{l}\text { ARTÍCULO } \\
X I\end{array}$ & $\begin{array}{l}\text { Recent Progress in } \\
\text { Modeling Biome- } \\
\text { Climate Interactions in } \\
\text { Amazônia. }\end{array}$ & $\begin{array}{l}\text { Nobre, 2004, } \\
\text { Brasil. }\end{array}$ & $\begin{array}{l}\text { La investigación tuvo como } \\
\text { objetivo investigar la relación } \\
\text { entre la deforestación } \\
\text { histórica y la precipitación a } \\
\text { diferentes escalas } \\
\text { geográficas en la Amazonía } \\
\text { del sur de Brasil } \\
\text { (SBA).También se evaluaron } \\
\text { los impactos de los } \\
\text { escenarios de políticas de } \\
\text { deforestación en la } \\
\text { agricultura de la región. }\end{array}$ \\
\hline $\begin{array}{l}\text { ARTÍCULO } \\
\text { XII }\end{array}$ & $\begin{array}{l}\text { Population Growth } \\
\text { and Net Migration in } \\
\text { the Brazilian Legal } \\
\text { Amazon, 1970- 1996, } \\
\text { em WOOD e PORRO. }\end{array}$ & $\begin{array}{l}\text { Perz, 2002, } \\
\text { Estados } \\
\text { Unidos. }\end{array}$ & $\begin{array}{l}\text { El artículo se basó en datos } \\
\text { del censo demográfico } \\
\text { brasileño para mostrar cómo } \\
\text { crecieron las poblaciones en } \\
\text { la Amazonía entre } 1970 \text { y } \\
\text { 1996, lo que llevó a la }\end{array}$ \\
\hline
\end{tabular}

RC: 104516

Lien d'accès: https://www.nucleodoconhecimento.com.br/sante/emergence-de-maladies 


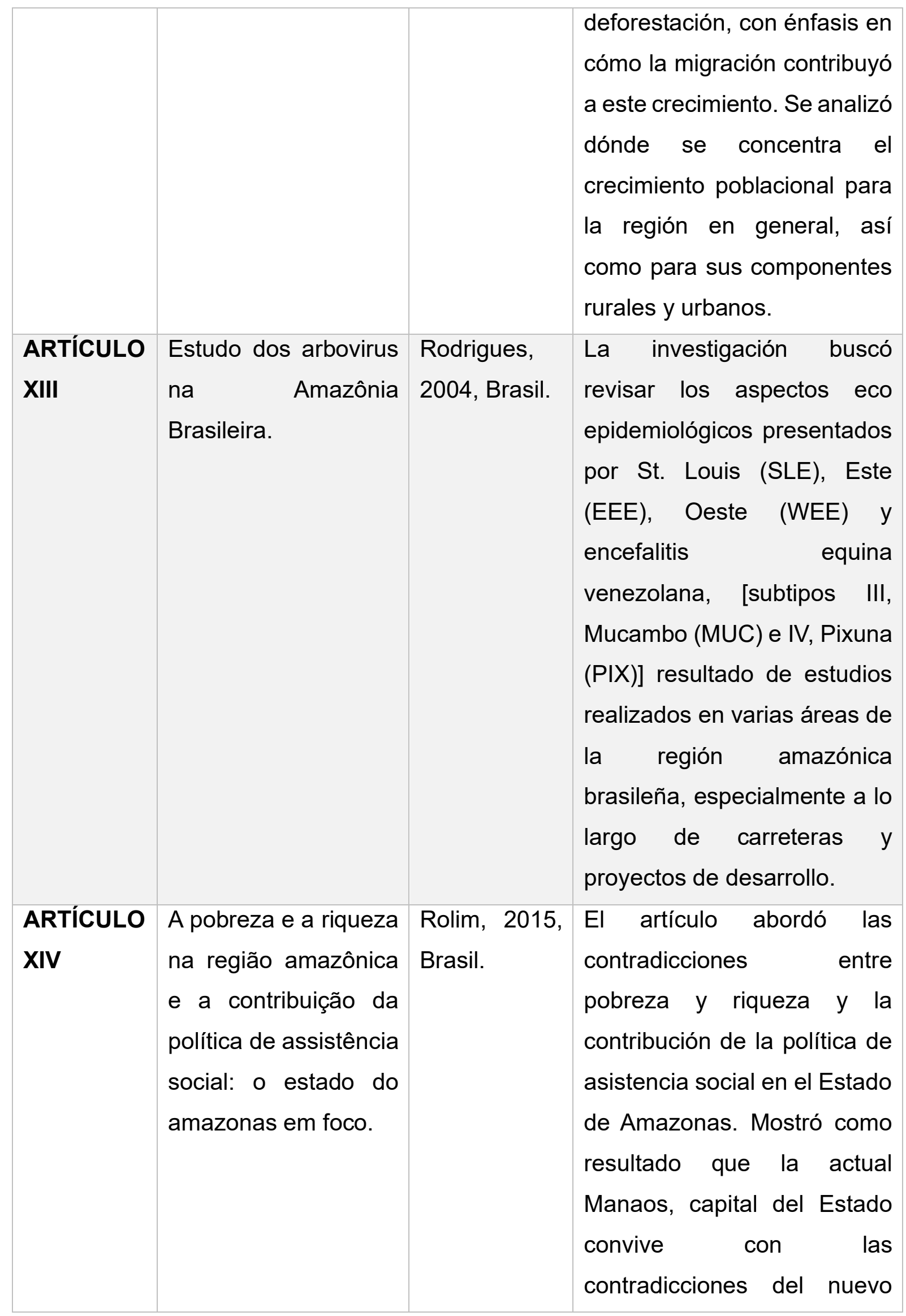

RC: 104516

Lien d'accès: https://www.nucleodoconhecimento.com.br/sante/emergence-de-maladies 


\begin{tabular}{|c|c|c|c|}
\hline & & & $\begin{array}{l}\text { orden capitalista donde se } \\
\text { visualiza la yuxtaposición } \\
\text { entre riqueza y pobreza. }\end{array}$ \\
\hline $\begin{array}{l}\text { ARTÍ́CULO } \\
\text { XV }\end{array}$ & $\begin{array}{l}\text { Modelo de } \\
\text { organização de } \\
\text { indicadores para } \\
\text { operacionalização } \\
\text { dos determinantes } \\
\text { socioambientais da } \\
\text { saúde. }\end{array}$ & $\begin{array}{l}\text { Sobral e } \\
\text { Freitas, 2010, } \\
\text { Brasil. }\end{array}$ & 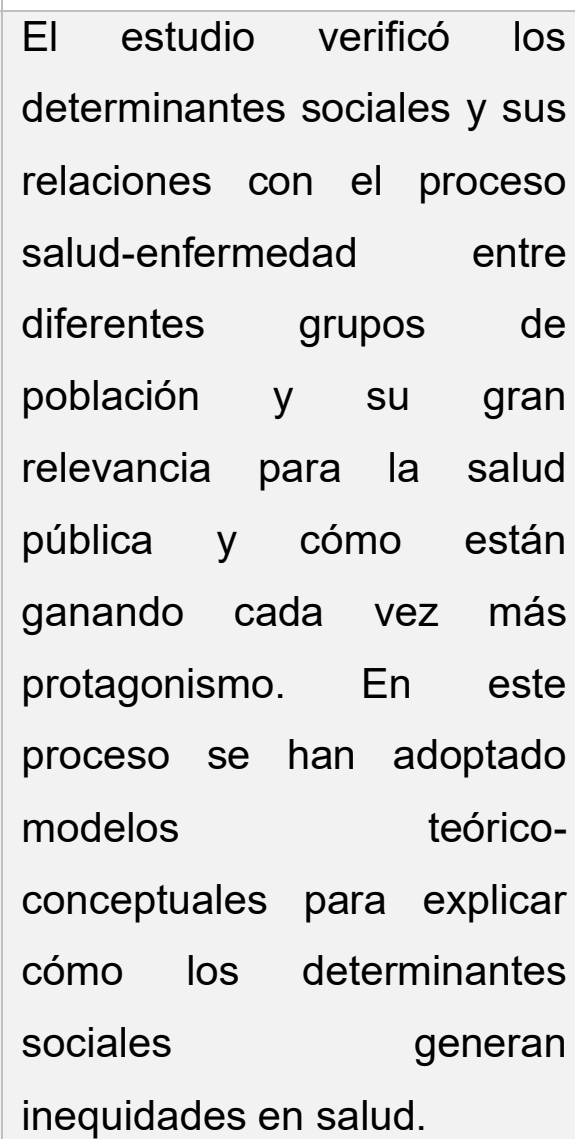 \\
\hline $\begin{array}{l}\text { ARTÍCULO } \\
\text { XVI }\end{array}$ & $\begin{array}{l}\text { Saúde ambiental e } \\
\text { desenvolvimento na } \\
\text { Amazônia legal: } \\
\text { indicadores } \\
\text { socioeconômicos, } \\
\text { ambientais e } \\
\text { sanitários, desafios e } \\
\text { perspectivas. }\end{array}$ & $\begin{array}{l}\text { Viana et al., } \\
\text { 2016, Brasil. }\end{array}$ & $\begin{array}{l}\text { El artículo buscó discutir los } \\
\text { procesos de determinación } \\
\text { socioambiental en los } \\
\text { estados que conforman la } \\
\text { Amazonía Legal, a partir del } \\
\text { análisis de indicadores } \\
\text { socioeconómicos, } \\
\text { ambientales y de salud. }\end{array}$ \\
\hline $\begin{array}{l}\text { ARTÍCULO } \\
\text { XVII }\end{array}$ & $\begin{array}{l}\text { Saúde, ambiente e } \\
\text { desenvolvimento } \\
\text { econômico na } \\
\text { Amazônia. }\end{array}$ & $\begin{array}{l}\text { Vincentin e } \\
\text { Minayo, } \\
\text { 2003, Brasil. }\end{array}$ & $\begin{array}{l}\text { El estudio analizó el proceso } \\
\text { de transformación del patrón } \\
\text { epidemiológico de una } \\
\text { población amazónica } \\
\text { constituida para trabajar en el }\end{array}$ \\
\hline
\end{tabular}

RC: 104516

Lien d'accès: https://www.nucleodoconhecimento.com.br/sante/emergence-de-maladies 


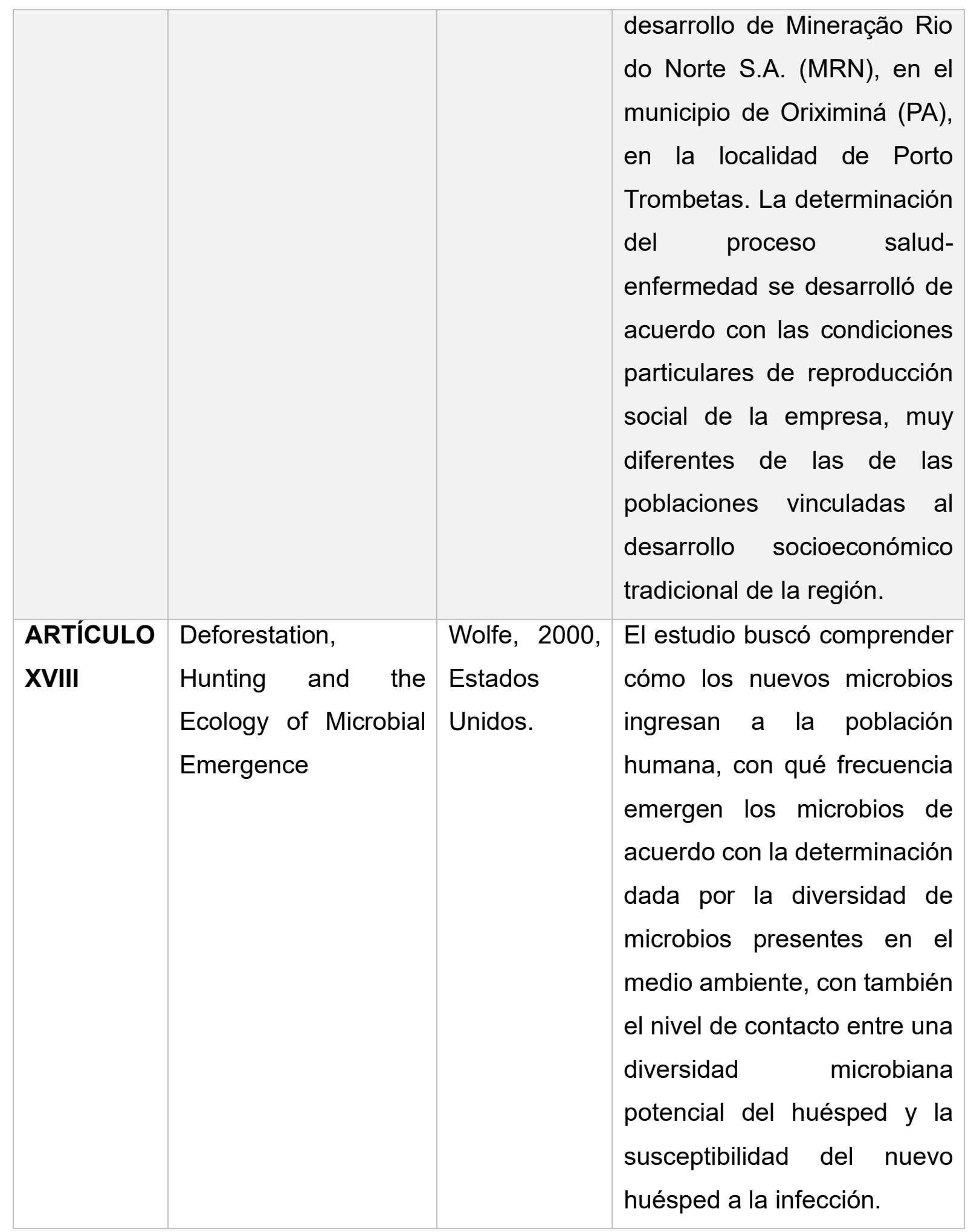

Fuente: autores propios.

Entre los artículos elegidos, la gran mayoría de los estudios mostraron que los factores ambientales y sociológicos que contribuyen a la aparición de enfermedades en la RC: 104516

Lien d'accès: https://www.nucleodoconhecimento.com.br/sante/emergence-de-maladies 
región amazónica desde 1970, como se muestra en el artículo "Chagas disease and globalization of the Amazon" del autor Briceño-León (2007), conforman esta cuenca histórica. Este cambio en la epidemiología de las enfermedades en la región amazónica puede explicarse por los cambios ambientales y sociales en los últimos 30 años, según explicó el autor.

Los autores Browder y Godfrey (2017), Browder y Godfrey (2017), Cardoso (2015), Carrapato et al. (2017), Confalonieri (2000), Confalonieri (2005), Medeiros (2014), Nobre (2004), Perz (2002), Sobral e Freitas (2010) y Viana et al. (2016) en su investigación publicada, en resumen, mostró la relación de los determinantes sociales y ambientales y su conexión intrínseca con el proceso salud-enfermedad de diferentes poblaciones, su relevancia significativa para la salud pública y cómo se hicieron más prominentes. En este proceso de años de historias que la Amazonía ha venido enfrentando con cambios sociales y espacio físico y territorial, y como consecuencias el agravamiento de enfermedades concentradas en esta región.

\section{REVISIÓN Y DISCUSIÓN DE LA LITERATURA}

\subsection{ASPECTOS REGIONALES}

Un fenómeno muy importante en el medio ambiente amazónico es la interacción entre la atmósfera y los bosques, que juegan un papel importante en la producción de lluvias en la región. El clima es generalmente cálido y húmedo y la temperatura no cambia mucho, siendo un importante regulador de los procesos biológicos, especialmente los relacionados con enfermedades infecciosas (NOBRE, 2004).

Otro aspecto biofísico relacionado tiene que ver con la biodiversidad, especialmente con la diversidad animal. Algunos procesos de infección focal en esta área, especialmente los procesos de infección viral, dependen de insectos chupadores de sangre (vectores) para su transmisión (arbovirus). Cuanto mayor es la diversidad de especies animales (vertebrados e invertebrados) en un área determinada, mayor es el riesgo de un nuevo proceso de infección por Wolfe (2000). Para ejemplificar este 
punto, se identificaron alrededor de 196 arbovirus en la Amazonía, 32 de los cuales pueden infectar a los humanos (RODRIGUES, 2004).

Nobre (2004) señala que en relación con la sociedad amazónica, se pueden destacar los siguientes aspectos demográficos importantes:

Yo. Baja densidad de población regional (aproximadamente 20 millones de habitantes en un área de 3,5 millones de kilómetros cuadrados) (INPE, 2013; IBGE, 2013);

II. la alta tasa de crecimiento proporcionada por la inmigración en los últimos 30 años. También hay una fuerte migración interregional (PERZ, 2002);

III. las ciudades están muy concentradas, especialmente en las capitales. Aproximadamente el $70 \%$ de la población amazónica vive en ciudades grandes y pequeñas. Es característica la aparición rápida, espontánea y desordenada de muchos de estos pequeños asentamientos urbanos sin la infraestructura de saneamiento necesaria (BROWDER y GODFREY, 2017);

IV. hay un gran número de poblaciones tradicionales (indios, ribereños, recolectores de caucho, etc.). que viven del extractivismo y la pequeña agricultura (INPE, 2013; IBGE, 2013).

Otra característica regional importante se refiere a las prácticas de uso de la tierra y sus impactos ambientales y sociales. Algunas de estas prácticas producen cambios ambientales que presentan riesgos para la salud. Es el caso de la contaminación por mercurio en las minas; debido a la falta de infraestructura de saneamiento en las zonas urbanas, el agua está contaminada por microorganismos; el humo de la quema después de la deforestación causa problemas respiratorios; cambios locales en el ciclo hidrológico que producen criaderos de mosquitos, para enumerar sólo unos pocos efectos más directos (CONFALONIERI, 2000).

También debe recordarse que los conflictos sociales, por ejemplo, la tenencia de la tierra conducen a la violencia y los conflictos culturales, y el impacto drástico de los pueblos indígenas aislados y la introducción de agentes microbianos previamente

$\mathrm{RC}: 104516$

Lien d'accès: https://www.nucleodoconhecimento.com.br/sante/emergence-de-maladies 
desconocidos por estas comunidades, proporcionando la aparición de nuevas enfermedades y aumentando el número de casos de las existentes en la región (RODRIGUES, 2004).

El trasfondo común de estos eventos es que las políticas de desarrollo y uso de recursos ignoran el potencial regional, olvidando la resiliencia ambiental y la sostenibilidad ecológica de la Amazonía, no conducen a la cultura tradicional o la distribución del ingreso y no se preocupan por los riesgos para la salud (CONFALONIERI, 2000).

En la práctica, esto está sucediendo tanto por iniciativa del gobierno, a través de la implementación de grandes proyectos de infraestructura (carreteras, centrales hidroeléctricas, etc.). e industria (minería comercial), fomentando la ganadería (y la deforestación), así como a través de actividades extractivas espontáneas como la minería, la pesca, la tala y otras (BRICEÑO-LEÓN, 2007; VICENTIN y MINAYO, 2003; FREITAS y GIATTI, 2009).

\subsection{INDICADORES SOCIOAMBIENTALES}

En el espacio que rodea las partes sur y este de la Amazonía (estados de Rondônia, Mato Grosso, Tocantins, Maranhão y Pará), los brotes de incendios, la deforestación acumulada y la construcción de carreteras, así como las áreas de cultivo e intensificación del consumo de pesticidas se han intensificado en los últimos años (INPE, 2013; IBGE, 2013).

Estos recursos fueron llamados por Nobre (2004) "arco de fuego". En cuanto al consumo de plaguicidas, las tasas alcanzadas con estas sustancias en la región fueron expresivas, representando el $18 \%$ del total nacional. Según datos publicados en 2009 por el Instituto Brasileño de Geografía y Estadística (IBGE), el estado de Mato Grosso alcanzó la tasa nacional más alta de uso de pesticidas por hectárea de área cultivada en la Amazonía Legal (IBGE, 2013).

Según los indicadores examinados, los datos de aumento de tierras cultivables y pastizales en áreas naturales y deforestación acumulada en la Amazonía alcanzaron RC: 104516

Lien d'accès: https://www.nucleodoconhecimento.com.br/sante/emergence-de-maladies 
las tasas más altas en el mismo 'arco de fuego', con clara convergencia entre la expansión de la agricultura extensiva y la deforestación junto con la ocurrencia de incendios y el uso de pesticidas (CARDOSO, 2015).

Siguiendo el razonamiento y rescate de Confalonieri (2005), es posible mostrar cuánto experimentan las sociedades amazónicas dificultades para acceder a la salud y, en consecuencia, la falta de atención y enfermedades. El autor llama la atención sobre factores como: baja densidad demográfica regional, crecimiento significativo de la migración, incluidas las migraciones interregionales, alta concentración de ciudades, especialmente en las capitales, con falta de infraestructura sanitaria y un gran número de poblaciones tradicionales, perjudica los determinantes sociales en la Amazonía, causando graves problemas de salud.

Para los autores Medeiros et al. (2014) también se discutió la situación de las condiciones de trabajo en las áreas de paisaje natural y antrópico de la Amazonía. Esto se debe a que las condiciones y el entorno de trabajo son determinantes de las enfermedades de salud ocupacional y se clasifican como enfermedades no transmisibles. Por lo tanto, la salud del trabajador se conceptualiza como:

Um campo de saúde pública que tem como objeto de estudo e intervenção as relações produção-consumo e o processo saúde-doença. Neste campo, o trabalho é considerado como eixo organizador da vida social, espaço de dominação e resistência dos trabalhadores e determinantes das condições de vida e saúde das pessoas (BRASIL, 2018, p. 136).

El trabajo es un determinante importante de la vida y la salud de las personas. Sabiendo que existen muchas actividades, relaciones y condiciones de servicio diferentes en la región amazónica, es importante que los profesionales de la salud promuevan, prevengan y diagnostiquen enfermedades relacionadas con las tareas que se realizan en la región amazónica (CARDOSO, 2015).

En estas actividades profesionales muy específicas de la Amazonía, que son raras en otras partes de Brasil, se puede mencionar: extractivismo vegetal - profesión, que RC: 104516 
incluye, entre otros, açaí extractivo, compactador de caucho, recolector de castaña, pilotos de barcos de transporte de pasajeros, prospectores, pescadores y muchos más. También se percibe que las relaciones laborales altamente degradantes, que en muchos lugares pueden considerarse análogas a la esclavitud, son bastante evidentes en la región debido a la dificultad de controlar la gran expansión de la tierra en la Amazonía.

Debido a que es un área rica en minerales, los buscadores se asientan en esta región y en áreas protegidas donde la minería está prohibida. Como resultado de la entrada en las áreas forestales, tanto los prospectores como los extractores de nueces de Brasil y los recolectores de caucho se ven afectados por enfermedades bacterianas transmitidas por vectores o protozoos no transmisibles y protozoos que existen en el bosque.

Entre ellos, se puede mencionar la enfermedad de Chagas, la malaria, la leishmaniasis y muchas enfermedades virales en la región. Por lo tanto, dado el gran número de estos profesionales y los riesgos que enfrentan, el sistema de salud debe dirigir acciones para que las enfermedades directa o indirectamente relacionadas con el trabajo no se conviertan en un factor de aumento de la morbilidad y mortalidad en la región amazónica (CARDOSO, 2015).

Es importante destacar que el debate sobre el trabajo de la Amazonía y su alcance es muy rico, ya que también muestra que la Amazonía es un lugar que aún es un reciente de investigación específica y políticas de salud pública sobre condiciones de vida, salud-enfermedad y trabajo en la región (BENSABATH y SOARES, 2016).

\subsection{INDICADORES EPIDEMIOLÓGICOS REGIONALES}

En relación con los indicadores epidemiológicos regionales, existen algunas diferencias importantes entre la región amazónica y el resto del país. En 1996, la tasa de hospitalizaciones por enfermedades infecciosas y parasitarias fue muy alta $(12,7 \%)$, muy superior a la media nacional $(8,3 \%)$. Aunque la incidencia de estas 
lesiones ha disminuido en la Amazonía, la tasa de mortalidad por estas enfermedades no ha sido la misma (SUDAM, 2000).

Esta región alberga la gran mayoría de los casos de malaria en el país (98\%), aproximadamente el $35 \%$ de los casos de lepra en 2016, y la segunda mayor incidencia de tuberculosis entre todas las regiones del país. También es un área altamente endémica de hepatitis viral con brotes frecuentes, especialmente en la Amazonía occidental (BENSABATH y SOARES, 2016).

Cabe destacar que debido a las condiciones geográficas de la región, así como al proceso de urbanización y falta de saneamiento básico contribuyeron al proceso de enfermedades que resurgieron en la Amazonía, como el cólera en 1991 y el dengue a mediados de la década de 1990, además de estas, otras enfermedades también causan grandes cantidades de muertes en la población de la región como la malaria, Leishmaniasis tegumentaria americana infecciones parasitarias y especialmente tuberculosis (VIANA et al., 2016).

La literatura también revela la relación desproporcionada entre la mejora de la calidad de vida y la degradación ambiental, es decir, el desarrollo avanza de manera insostenible, y destaca la importancia de considerar el costo de las enfermedades causadas por factores ambientales, que ha aumentado en la región amazónica (MEDEIROS et al., 2014).

Carrapato; Correa y García (2017) vincularon de inmediato la calidad de vida con la salud. Los autores destacan que, además de los modelos biomédicos, existen algunos factores decisivos que han afectado o afectarán decisivamente a la salud personal. Por lo tanto, estos determinantes actualmente tienen un mayor impacto en la salud que los que actualmente cubre la medicina. Destacaron la existencia de tres determinantes: el medio ambiente, la economía y la sociedad, para ellos los determinantes sociales son en gran parte responsables de la injusticia del acceso a la atención médica. 


\section{CONCLUSIÓN}

Con base en la pregunta principal del estudio, es posible inferir que los principales factores ambientales que contribuyen a la aparición de enfermedades en la región amazónica son la deforestación, la quema, la contaminación de los ríos, el proceso de urbanización y la agroindustria, concluyendo así que la discusión sobre el estudio finalmente identificó algunos temas que son responsables del número de enfermedades en la Amazonía, donde se deben, por ejemplo, considerar los aspectos geográficos, étnicos, culturales y políticos de la salud en la región amazónica. Se denota que existe una falta de políticas públicas que atiendan la demanda de enfermedades de salud relacionadas con los indicadores socioambientales de la región, así como la necesidad de realizar más estudios e investigaciones para conocer las adversidades que enfrentan estas poblaciones.

También es de destacar que los principales factores ambientales que contribuyen a la aparición de enfermedades en la región amazónica son la deforestación, la quema, la contaminación fluvial, el proceso de urbanización y la agroindustria, es decir, todo desde la acción del hombre.

Sin embargo, el estudio también muestra que desde la perspectiva de los indicadores ambientales de salud y enfermedad, el espacio amazónico formado por la interacción del sistema socioecológico tiene características distintas de otras partes del país. Esta diferencia es causada por la base ecológica natural y sus formas de ocupación y desarrollo.

\section{REFERENCIAS}

BENSABATH, G. y SOARES, M. C. P. La evolución del conocimiento sobre las hepatitis virales en la región amazónica: de la epidemiología y la etiología a la prevención. Rev. Soc. Bras. Med. Trop., 37(supl. II):14-36, 2016.

BRASIL. Ministério da Saúde. Secretaria de Atenção à Saúde. Secretaria de Vigilância em Saúde. Saúde do trabalhador e da trabalhadora [recurso eletrônico]. Cadernos de Atenção Básica, n. 41 - Brasília: Ministério da Saúde, 2018. 136 p.: il. RC: 104516 
BRICEÑO-LEÓN, R. Chagas disease and globalization of the Amazon. Cadernos de Saúde Pública, Rio de Janeiro, v. 23, n. 53, p. 33-40, 2007. Suplemento 1.

BROWDER, J. O. e GODFREY, B. J. Rainforest Cities. Urbanization, Development and Colonization of the Brazilian Amazon. Columbia University Press, New York, 2017, $429 \mathrm{pp}$.

CAÑETE, T. M. R..; RAVENA-CAÑETE, V. Populações Tradicionais Amazônicas: revisando conceitos. In: V Encontro Nacional da Associação Nacional de PósGraduação e Pesquisa em Ambiente e Sociedade, 2010, Florianópolis Anais, V Encontro Nacional da Associação Nacional de Pós-Graduação e Pesquisa em Ambiente e Sociedade, 2010.

CARDOSO, A. C. M. O trabalho como determinante do processo saúde-doença. Tempo soc., São Paulo, v. 27, n. 1, p. 73-93, junho de 2015.

CARRAPATO, P.; CORREIA, P.; GARCIA, B. Determinante da saúde no Brasil: a procura da equidade na saúde. Saúde soc., São Paulo, v. 26, n. 3, p. 676-689, Sept. 2017.

CONFALONIERI, U. E. C. (org.). The Health of Indigenous Peoples in the Brazilian Amazon. Background paper for the World Bank. Royal Tropical Institute, Amsterdam, 2000, $218 \mathrm{pp}$.

CONFALONIERI, U. E. C. Saúde na Amazônia: um modelo conceitual para a análise de paisagens e doenças. Estud. av., São Paulo, v. 19, n. 53, p. 221-236, Apr. 2005.

FREITAS, C. M.; GIATTI, L. L. Indicadores de sustentabilidade ambiental e de saúde na Amazônia Legal. Cadernos de Saúde Pública, Rio de Janeiro, v. 25, n. 6 , p. 1251-1266, 2009.

IBGE - INSTITUTO BRASILEIRO DE GEOGRAFIA E ESTATíSTICA. Contas nacionais: 2011. Rio de Janeiro, RJ, 2013. 
INPE - Instituto Nacional De Pesquisas Espaciais. Monitoramento da floresta amazônica brasileira por satélite: 1988/2011. 2013.

MEDEIROS, M. S. de et al. Custo das doenças atribuíveis a fatores ambientais na cidade de Manaus, Amazonas, Brasil. Ciênc. Saúde coletiva [online]. 2014, vol.19, n.2, pp.599-608. 2014.

NOBRE, C. A. Recent Progress in Modeling Biome-Climate Interactions in Amazônia. Resumo 19.2. Anais III Conf. Cient.do LBA, 27-29 de julho, 2004. Brasília, DF, Cd-Rom.

PERZ, S. Population Growth and Net Migration in the Brazilian Legal Amazon, 1970- 1996, em WOOD e PORRO. Deforestation and Land Use in the Amazon. Gainesville, University Press of Florida, 2002, pp. 95-106.

RODRIGUES, S. G. Estudo dos arbovirus na Amazônia Brasileira. Em: Resumos do III Simpósio Internacional sobre Arbovirus nos Trópicos e Febres Hemorrágicas, Belém, Pará, 30/11 - 3/12, 2004. Instituto Evandro Chagas, 2004.

ROLIM, D. C. A pobreza e a riqueza na região amazônica e a contribuição da política de assistência social: o estado do amazonas em foco. VII Jornada Internacional de Políticas Públicas. Programa de Pós-graduação em políticas públicas. Universidade Federal do Maranhão, 2015.

SOBRAL, A.; FREITAS, C. M. Modelo de organização de indicadores para operacionalização dos determinantes socioambientais da saúde. Saúde e Sociedade, São Paulo, v. 19, n. 1, p. 35-47. 2010.

SUDAM. Diagnóstico e cenarização macrossocial da Amazônia legal: perfil da saúde na Amazônia legal e o contexto brasileiro. Belém, Pnud, 2000, 54 p.

VIANA, Rosana Lima; FREITAS, Carlos Machado de; GIATTI, Leandro Luiz. Saúde ambiental e desenvolvimento na Amazônia legal: indicadores socioeconômicos, ambientais e sanitários, desafios e perspectivas. Saúde e Sociedade, v. 25, p. 233246, 2016.

RC: 104516

Lien d'accès: https://www.nucleodoconhecimento.com.br/sante/emergence-de-maladies 
VICENTIN, G.; MINAYO, C. G. Salud, medio ambiente y desarrollo económico en la Amazonía. Ciencia y Salud Pública, Río de Janeiro, v. 8, n. 4, p. 1069-1085, 2003.

WOLFE, N. Deforestación, caza y ecología de la emergencia microbiana. Cambio Global \& Um. Salud, 1(1):10-25, 2000.

Enviado: Diciembre de 2021.

Aprobado: Diciembre de 2021 